\title{
A Labelled Sequent-Calculus for Observation Logic
}

\author{
Olivier Brunet \\ Équipe EXMO - INRIA Rhône-Alpes \\ Zirst - 655 avenue de l'Europe \\ Montbonnot - 38334 Saint Ismier Cedex - France \\ E-mail: obruneteinrialpes.fr
}

\begin{abstract}
We investigate observation logic, an intuitionnistic modal logic designed for reasoning about approximation and multiple contexts, and propose a sequent-calculus formulation of this logic. Due to the validy of an axiom (called T2) which is a weakening of $\mathbf{T}$, one needs an adaptation of the usual sequent-calculus formalism in order to have some classical properties of sequent calculus, such as cut elimination and the subformula property.

To solve this problem, we propose to assign to each proposition inside a proof a label, carrying some context information, and show the validity of some expected properties and manipulations in this framework.
\end{abstract}

\section{Introduction}

Observation logic $[4,3]$ is a formalization of the way information behaves in a partial-observation context, when all knowledge comes from some possibly partial observation. This logic originated as an axiomatization of a validity predicate defined over some general algebraic structures, called representation systems. Those structures have been designed as an attempt to provide a general construction which embodies the notion of approximate representation (since our observations, being partial, can be seen as a partial description of its state), but without having the studied or observed system explicitly represented.

This provides a new and general approach to the problem of reasoning about approximation $[12,13,19,17]$ and about multiple contexts and theories $[18,16,1]$. In particular, observation logic is a modal intuitionnistic logic with a collection of modal operators (denoted $K_{i}$ ) which can all be associated to a partial way to consider informations about a system. Thus, those operators each correspond to an approximation method, and can all be seen as a partial observation method too. They behave in an $\mathbf{S} 4$ way, but with a few adaptions. The most important one is that axiom $\mathbf{T}: K_{i} \varphi \rightarrow \varphi$ is not valid, but weaker versions of this axiom are valid, namely $\mathbf{T} 2: K_{i} K_{j} \varphi \rightarrow K_{j} \varphi$ and LT : $K_{i}\left(K_{i} \varphi \rightarrow \varphi\right)$. If the latter is just a characteristic of the way knowledge behaves internally, the former is very important, and is a cornerstone of the theory, as it permits to relate knowledge and information between different contexts.

In the present paper, we present a sequent calculus for the logic, and show some of its properties, and some proofs manipulations. The main characteristic of this calculus is the use of labels (represented by finite words over the set of indexes) for propositions, which permits to deal with axiom T2, and have the subformula property verified. We also show expected results such as that of cut-elimination.

\section{Observation Logic}

In this section, we provide an overview of representation systems and observation logic. Further developments can be found in $[4,3]$.

\subsection{Representation Systems}

Representation systems were introduced as an attempt to provide a general algebraic framework for formalizing the notions of partial observation and partial description. Intuitively, one may define an approximation process using the following structures : first, the system to be studied and approximated can be represented by a poset $\left\langle P_{\mathcal{S}}, \leq_{\mathcal{S}}\right\rangle$ which elements can be seen for instance as sets of possible states, in a Kripke's possible worlds approach [15], where the partial order $\leq_{\mathcal{S}}$ is such that if $d_{1} \leq_{\mathcal{S}} d_{2}$, then $d_{1}$ is a more precise description of the state of the system than $d_{2}$ (in 
terms of possible worlds, the set of possible states associated to $d_{1}$ is included in the set of possible states associated to $d_{2}$ ). The result of the approximation can also be formalized using a poset $\left\langle P_{\mathcal{A}}, \leq_{\mathcal{A}}\right\rangle$. Then, the approximation relation between those two posets can be defined as a Galois surjection $[20,21,2,7,6]$ :

$$
\left\langle P_{\mathcal{S}}, \leq_{\mathcal{S}}\right\rangle \underset{\alpha}{\stackrel{\gamma}{\leftrightarrows}}\left\langle P_{\mathcal{A}}, \leq_{\mathcal{A}}\right\rangle
$$

This provide a natural way to express approximations, as given an element $d$ of $P_{\mathcal{S}}$ corresponding to a set of possible states of the system, one associates the element $\alpha(d)$ of $P_{\mathcal{A}}$ which can be seen as an approximate description of the state of the system. In particular, it can be seen as an approximation of $d$, since from the definition of Galois surjections, one has :

$$
d \leq \mathcal{S} \gamma \circ \alpha(d) \quad \alpha(d)=\alpha \circ \gamma \circ \alpha(d)
$$

This construction can be generalized by considering a collection of approximation methods (indexed by elements $i$ of a set $\mathcal{I})$, each defined by a poset $\left\langle P_{i}, \leq_{i}\right\rangle$ and a Galois surjection $\left\langle\alpha_{i}, \gamma_{i}\right\rangle$.

From this, it is possible to introduce some "transformation functions" relating the different approximate posets $P_{i}$ by defining $f_{i \mid j}=\alpha_{i} \circ \gamma_{j}$. With these functions, it is possible to express relationships between the different possible approximations of a given system, without making explicitly reference to this system. Moreover, considerations about the properties verified by Galois surjections can be used to identify properties verified by the $f_{i \mid j}$ functions in our formalism, which we will use as a characterization of our "transformation functions". This leads to the definition of representation systems.

\section{Definition 1 (Representation System)}

A representation system is a triplet :

$$
\left\langle\mathcal{I},\left\{\left\langle P_{i}, \leq_{i}\right\rangle\right\}_{i},\left\{f_{i \mid j}\right\}\right\rangle
$$

where $\mathcal{I}$ is a set of indexes, for each $i \in \mathcal{I},\left\langle P_{i}, \leq_{i}\right\rangle$ is a poset call a representation, and such that the functions $f_{i \mid j}: P_{j} \rightarrow P_{i}$ verify :

$$
\begin{array}{lr}
f_{i \mid i}=\operatorname{id}_{i} & \text { Identity } \\
d \leq_{j} d^{\prime} \Rightarrow f_{i \mid j}(d) \leq_{i} f_{i \mid j}\left(d^{\prime}\right) & \text { Monotony } \\
f_{i \mid k} \leq f_{i \mid j} \circ f_{j \mid k} & \text { Composition }
\end{array}
$$

Actually, this definition is sufficient for ensuring that all the representations can be considered as approximations of a single system, since given a representation system $\mathcal{S}$, it is possible to build a poset $P_{\mathcal{S}}$ and a collection of Galois surjections $\left\langle\alpha_{i}, \gamma_{i}\right\rangle$ from $P_{\mathcal{S}}$ to $P_{i}$ such that $f_{i \mid j}=\alpha_{i} \circ \gamma_{j}$.
Thus, in our formalism, one manipulates approximations and partial descriptions of a given system, but the system itself is not present explicitly in the algebraic structure and is only present through the relationship that exist between the different partial representations.

In order to provide a general and flexible way to study the way knowledge and information behaves in this formalism, we will now introduce a logical formalization of those structures.

\subsection{Logical Translation}

Let us first define our langage $\mathcal{L}_{\mathcal{I}, \Psi}$ by the grammar :

$$
P=A P|\perp| P \vee P|P \wedge P| P \rightarrow P \mid K_{i} P
$$

In this definition, a term $A P$ stands for an element $\psi$ in the set of atomic propositions $\Psi$, and in a term of the form $K_{i} P$, the indice $i$ is an element of $\mathcal{I}$.

To relate $\mathcal{L}_{\mathcal{I}, \Psi}$ and a representation system $\mathcal{S}$ indexed by $\mathcal{I}$, we will define a collection of interpretation functions $\llbracket \cdot \rrbracket_{i}: \mathcal{L}_{\mathcal{I}, \Psi} \rightarrow \wp^{\downarrow}\left(P_{i}\right)$ (where $\wp^{\downarrow}\left(P_{i}\right)$ stands for the set of ideals, i.e. of downward-closed subsets of $P_{i}$ ). Given a proposition $\varphi$, the ideal $\llbracket \varphi \rrbracket_{i}$ corresponds to the set of elements of $P_{i}$ which, seen as partial descriptions of the state of the system, provide enough informations in order to prove that property $\varphi$ actually holds. This set has to be an ideal, since if a element $d$ is in this set, so will be any element $d^{\prime} \leq_{i} d$, since $d^{\prime}$ provides more information that $d$.

This function is defined inductively from the structure of terms. For atomic propositions, one has to provide an atomic interpretation $\nu_{i}: \Psi \rightarrow \wp^{\downarrow}\left(P_{i}\right)$. For the classical connector, the interpretation corresponds to intuitionnistic logic, since all propositions are interpreted as ideals of a poset. For modal connector, the interpretation $\llbracket K_{j} \varphi \rrbracket_{i}$ relies on the use of the transformation functions $f_{i \mid j}$, since it is the set of elements of $P_{i}$ which, after transformation in $P_{j}$ by $f_{j \mid i}$, lie in the interpretation $\llbracket \varphi \rrbracket_{j}$, so that $\llbracket K_{j} \varphi \rrbracket_{i}=\left\{d \mid f_{j \mid i}(d) \in \llbracket \varphi \rrbracket_{j}\right\}$. The definition of $\llbracket \cdot \rrbracket_{i}$ is summarized in figure 1 , where the explicit reference to a representation system $\mathcal{S}$ and an atomic interpretation $\nu$ is omitted.

With this interpretation function, it is possible to define a validity notion, so as to identify which propositions of $\mathcal{L}_{\mathcal{I}, \Psi}$ do properly correspond to the behavior of information in our partial description approach.

Definition 2 (Validity)

A proposition $\varphi$ is valid for a representation system $\mathcal{S}$ and an atomic interpretation $\nu$ over $\mathcal{S}$ (which we denote $\langle\mathcal{S}, \nu\rangle \models \mathcal{S} \varphi)$ if and only if $\forall i, \llbracket \varphi \rrbracket_{\mathcal{S}, \nu, i}=P_{i}$. 


$$
\begin{aligned}
\llbracket \psi \rrbracket_{i} & =\nu_{i}(\psi) \quad \psi \in \Psi \\
\llbracket \varphi \vee \psi \rrbracket_{i} & =\llbracket \varphi \rrbracket_{i} \cup \llbracket \psi \rrbracket_{i} \\
\llbracket \varphi \wedge \psi \rrbracket_{i} & =\llbracket \varphi \rrbracket_{i} \cap \llbracket \psi \rrbracket_{i} \\
\llbracket \varphi \rightarrow \psi \rrbracket_{i} & =\left\{d \mid \forall d^{\prime} \leq d, d^{\prime} \in \llbracket \varphi \rrbracket_{i} \Rightarrow d^{\prime} \in \llbracket \psi \rrbracket\right\} \\
\llbracket \perp \rrbracket_{i} & =\emptyset_{i} \\
\llbracket K_{j} \varphi \rrbracket_{i} & =\left\{d \mid f_{j \mid i}(d) \in \llbracket \varphi \rrbracket_{j}\right\}
\end{aligned}
$$

\section{Figure 1. Interpretation Function}

A proposition $\varphi$ is valid for representation system if and only if it is valid for all representation systems and for all atomic interpretations for this representation system :

$$
\models_{\mathcal{S}} \varphi \Leftrightarrow \forall \mathcal{S}, \forall \nu,\langle\mathcal{S}, \nu\rangle \models_{\mathcal{S}} \varphi
$$

It has to be noted that, even though it is not clearly stated here, we are considering propositions and representation systems which relate to a given fixed index $\operatorname{set} \mathcal{I}$.

\subsection{Axiomatization}

We now provide an axiomatization of the notion of validity for representation systems by defining the logic OL, as the intuitionnistic logic $[14,8,23]$ together with the modal axioms and rules listed in figure 2 .

$$
\begin{array}{lr}
K_{i}(\varphi \rightarrow \psi) \rightarrow K_{i} \varphi \rightarrow K_{i} \psi & \mathbf{K} \\
K_{i} \varphi \rightarrow \neg K_{i} \neg \varphi & \mathbf{D} \\
K_{i} K_{j} \varphi \rightarrow K_{j} \varphi & \mathbf{T 2} \\
K_{i}\left(\varphi \leftrightarrow K_{i} \varphi\right) & \mathbf{L} \\
K_{i}(\varphi \vee \psi) \rightarrow K_{i} \varphi \vee K_{i} \psi & \mathbf{V} \\
& \\
\quad \frac{\vdash \varphi}{\vdash K_{i} \varphi} \text { Nec } & \frac{\forall i, \vdash K_{i} \varphi}{\vdash \varphi} \text { Univ }
\end{array}
$$

\section{Figure 2. Modal Axioms and Rules of OL}

A few comments can be done about OL. First, it can be seen as a multi-context reasoning logic. As exposed in $[16,5]$, axiom $\mathbf{K}$-modalities is a good candidate for defining formal systems for contexts. Moreover, axiom $\mathbf{T}$ $\left(K_{i} \varphi \rightarrow \varphi\right)$ is not valid, so that in our logic, facts inside a context need not be true, which emphasizes the fact that we are considering our contexts as approximations [18]. A weaker axiom (T2 : $K_{i} K_{j} \varphi \rightarrow K_{j} \varphi$ ) is valid, which, while not referring to "reality", allows to relate the different contexts, and thus reason with multiple contexts.
Moreover, in this logic, valid propositions are exactly the propositions which are valid in every context. The classical Nec rule tells that valid propositions are valid within every context. But in OL, the Univ-rule states the converse, that is if a proposition is valid in every context, then this proposition is considered as valid "objectively", with no reference to any context. In the case where there is a single context (where $\mathcal{I}$ is a singleton $\{\iota\}$ ), then the unique modal operator $K_{\iota}$ has no meaning, since one has in that case $\forall \varphi, \vdash \varphi \leftrightarrow K_{\iota} \varphi$. If the set $\mathcal{I}$ is finite, then this rule is equivalent to the axiom $\bigwedge_{i} K_{i} \varphi \rightarrow \varphi$. In the following, we will see that if $\mathcal{I}$ is infinite, this rule can actually be suppressed, since if an index $i$ does not appear in a formula $\varphi$, then proving the validity of $K_{i} \varphi$ is equivalent to proving that of $\varphi$ itself, and if $\mathcal{I}$ is infinite, it is always the case.

As expected, this logic is sound and complete with regards to representation systems, as we show in the following proposition :

\section{Proposition 1}

The logic OL is a sound and complete axiomatization of $\models_{\mathcal{S}}$.

Proof It is easy to check that $\models_{\mathcal{S}}$ is sound w.r.t. OL by checking that all its axioms are valid for representation systems.

The completeness proof can be done in a classical way using a canonical model $[8,23]$. The specific proof for OL can be found in $[4,3]$.

\section{Sequent Calculus}

\subsection{Words and Orders}

Before defining the rules that constitute our labeled sequent calculus, we will first introduce a few notations for dealing with those labels. Let $\mathcal{I}^{\star}$ denote the set of finite words over the alphabet $\mathcal{I}$. We also introduce the following notations : $\varepsilon$ is the empty word, $|\Lambda|$ the length of the word $\Lambda, \cdot$ the concatenation operation and given a word $\Lambda, \Lambda_{a \ldots b}$ is the word $\lambda_{a} \ldots \lambda_{b}$ with $a$ and $b$ being integers giving the range of the sub-word of $\Lambda$.

Intuitively, such words will be used to represent successions of $K_{i}$ operators. Terms will appear in sequents with labels, and a term of the form $[\varphi]_{\Lambda}$ in a sequent will be though of as equivalent to $K_{\Lambda_{n}} \ldots K_{\Lambda_{1}} \varphi$.

Now, since in OL, $K_{i} K_{i} \varphi$ and $K_{i} \varphi$ are equivalent (and more generally, $K_{\Lambda} K_{i} K_{i} K_{\Omega} \varphi$ and $K_{\Lambda} K_{i} K_{\Omega} \varphi$ are 
equivalent), it follows that an equivalence relation can be defined on words in order to capture the equivalence on the $K_{\Lambda}$. Thus, let $\simeq_{\mathcal{I}}$ denote the symmetric, transitive and reflexive closure of the relation $\sim$ defined by :

$$
\forall \Lambda, i, \Omega, \Lambda \cdot i \cdot i \cdot \Omega \sim \Lambda \cdot i \cdot \Omega
$$

From now on, we will identify the set of words $\mathcal{I}^{\star}$ with its classes of equivalence w.r.t. $\simeq_{\mathcal{I}}$, and those classes will by represented by words with no letter repetition. Let us now introduce two partial orders on $\mathcal{I}^{\star}$ (or more precisely, on its equivalence classes). The first one $(\leq)$ corresponds to the word inclusion ordering, while the other one $\left(\leq_{\star}\right)$ will be used to simulate the action of axiom $\mathbf{T} 2$.

\section{Definition 3 (Partial Orders on $\mathcal{I}^{\star}$ )}

Given two words $\Omega=\omega_{1} \ldots \omega_{n}$ and $\Lambda=\lambda_{1} \ldots \lambda_{m}, \Omega \leq \Lambda$ if and only if $\Omega$ is a sub-word of $\Lambda$, that is if and only if there exists an increasing function $\sigma:[1 \ldots n] \rightarrow[1 \ldots m]$ such that $\forall i, \omega_{i}=\lambda_{\sigma(i)}$.

Moreover, $\Omega \leq_{\star} \Lambda$ if and only if $\Omega \leq \Lambda$ and either $\Omega=\Lambda=\varepsilon$ or $\omega_{1}=\lambda_{1}$.

The partial order $\leq_{\star}$ has a very close relation to the $K_{i}$ operators' behavior with axiom $\mathbf{T} 2$, since one can show that if $K_{\Lambda} \varphi$ stands for $K_{\lambda_{n}} \ldots K_{\lambda_{1}} \varphi$, then one has :

$$
\Omega \leq_{\star} \Lambda \Leftrightarrow \forall \varphi, \vdash K_{\Lambda} \varphi \rightarrow K_{\Omega} \varphi
$$

This constitutes an alternate definition to $\leq_{\star}$, and in that case, $\leq$ can be defined in terms of $\leq_{\star}$, as :

$$
\Omega \leq \Lambda \Leftrightarrow \forall i \in \mathcal{I}, i \cdot \Omega \leq_{\star} i \cdot \Lambda
$$

This shows that how our two partial orders on $\mathcal{I}^{\star}$ can be closely related to the modal operators in OL.

A last point to be noted is that with our equivalence classes, our partial orders are such that given a word $\Lambda \in$ $\mathcal{I}^{\star}$, the sets $\{\Omega \mid \Omega \leq \Lambda\}$ and $\left\{\Omega \mid \Omega \leq_{\star} \Lambda\right\}$ are finite. Thus, they both are well-founded, which will be important in the induction order used for cut-elimination.

\subsection{Definition of the Calculus}

With those notations, we can now define our sequent calculus, as given in figure 3 . In this definition, each sequent is of the forme $\left[\gamma_{1}\right]_{\Lambda_{1}} \ldots\left[\gamma_{n}\right]_{\Lambda_{n}} \Vdash[\varphi]_{\Lambda}$, so that each proposition appearing in a sequent comes with an $\mathcal{I}$-word, called its localization. The use of localization permits to have the subformula property verified for $\Vdash$, since $K$-operators can be removed and replaced by the addition of an index in the localization. This can be illustrated in the following example :

$$
\begin{aligned}
& \begin{array}{cc}
\frac{j \leq_{\star} j \cdot i}{[\varphi]_{j \cdot i} \Vdash[\varphi]_{j}} & \frac{j \leq_{\star} j \cdot i}{[\varphi]_{j \cdot i} \Vdash[\varphi \vee \psi]_{j}} \\
\frac{[\psi]_{j \cdot i} \Vdash[\psi]_{j}}{\left[K_{j} \varphi\right]_{i} \Vdash\left[K_{j}(\varphi \vee \psi)\right]_{\varepsilon}} & \frac{[\psi]_{j \cdot i} \Vdash[\varphi \vee \psi]_{j}}{\left[K_{j} \psi\right]_{i} \Vdash\left[K_{j}(\varphi \vee \psi)\right]_{\varepsilon}}
\end{array} \\
& {\left[K_{j} \varphi \vee K_{j} \psi\right]_{i} \Vdash\left[K_{j}(\varphi \vee \psi)\right]_{\varepsilon}} \\
& \frac{\overline{\left[K_{i}\left(K_{j} \varphi \vee K_{j} \psi\right)\right]_{\varepsilon} \Vdash\left[K_{j}(\varphi \vee \psi)\right]_{\varepsilon}}}{\Vdash\left[K_{i}\left(K_{j} \varphi \vee K_{j} \psi\right) \rightarrow K_{j}(\varphi \vee \psi)\right]_{\varepsilon}}
\end{aligned}
$$

First, let us show that our sequent calculus is correct with regards to the observational logic $\mathrm{OL}$.

\section{Proposition 2}

The sequent calculus defined in figure 3 is sound and complete w.r.t. OL. More precisely, one has :

$$
\forall \varphi \in \mathcal{L}_{\mathcal{I}, \Psi}, \vdash \text { OL } \varphi \Leftrightarrow \emptyset \Vdash[\varphi]_{\varepsilon}
$$

Proof The $\Rightarrow$-implication can be easily proved by checking that all the axioms of OL can be proved in the sequentcalculus formalism.

To prove the $\Leftarrow$-implication, it suffice to show that all the rules are valid w.r.t. the following translation in terms of representation systems :

$$
\left[\gamma_{1}\right]_{\Lambda_{1}} \ldots\left[\gamma_{n}\right]_{\Lambda n} \Vdash[\varphi]_{\Lambda} \rightsquigarrow \forall i, \bigwedge_{j} \llbracket K_{\Lambda_{j}} \gamma_{j} \rrbracket_{i} \subseteq \llbracket K_{\Lambda} \varphi \rrbracket_{i}
$$

It follows from this that one has the implication :

$$
\forall \varphi, \emptyset \Vdash[\varphi]_{\varepsilon} \Rightarrow \models \mathcal{S} \varphi
$$

To conclude, one can remark that $\vdash_{\text {OL }}$ is sound and complete w.r.t. $\models_{\mathcal{S}}$.

Now, in order to use this sequent calculus efficiently for the search of proofs, this system cannot be used without precautions, especially due to the presence of two rules : first, the Cut-rule which may introduce new formulas, and the Univ-rule, which introduces new indexes. In the following section, we will investigate some proofs manipulations, and show that the Cut-rule is not necessary and can always be eliminated, and that the Univ-rule, though necessary, can always be used at most once as the first rule if all the indexes of $\mathcal{I}$ are present in the sequent, and it can be not used at all if an index does not appear.

\section{Proofs Manipulation}

\subsection{Label Manipulation On Proofs}

We will first study the way localisations behave inside proofs of the system $\Vdash$. From its rules, one can first remark 
that for any sequent $\Gamma \vdash[\varphi]_{\Lambda}$, all localized proposition $[\gamma]_{\Omega}$ in $\Gamma$ is such that $\Lambda \leq \Omega$. This result can be shown by induction on the height of the proof, since this property appears in the rules Axiom and $\perp$, and it is preserved by the application of the other rules (and it even appears explicitly for rule $K L)$.

Moreover, another property can be stated : given a proof $\Pi$ of a sequent $\Gamma \Vdash[\varphi]_{\Lambda}$, any sequent $\Delta \Vdash[\psi]_{\Omega}$ in $\Pi$ is such that $\Lambda \leq \Omega$. By combining those two properties, one gets the following result :

\section{Proposition 3}

Given a proof $\Pi$ of a sequent $\Gamma \vdash[\varphi]_{\Lambda}$, any term $[\psi]_{\Lambda^{\prime}}$ appearing in $\Pi$ is such that $\Lambda \leq \Lambda^{\prime}$.

This property suggests some ways to manipulate the localizations appearing in a proof. For instance, given a proof $\Pi$ of $\Gamma \vdash[\varphi]_{\Lambda}$, proposition 3 asserts that any localization $\Omega$ in $\Pi$ can be written as $\Omega_{1} \circ \Omega_{2}$ with $\Lambda \leq_{\star} \Omega_{2}$. The $\leq_{\star}$-relationship between $\Lambda$ and $\Omega_{2}$ in this case can be interpreted by the application of the Univ-rule which adds indexes on the right of $\Omega_{2}$, and of the Loc-rule which adds "garbage".

This means that one would want to "clean" a proof by replacing $\Omega$ by $\Omega_{1} \circ \Lambda$ for every localization $\Omega$ of $\Pi$. An even more interesting manipulation would be to replace those localizations by $\Omega_{1} \circ \Lambda^{\prime}$ for some $\Lambda^{\prime} \leq_{\star} \Lambda$. Such manipulation appears to be in fact necessary in one wants to have the cutelimination property for $\Vdash$, since if one has a proof of $\Pi$ of $\Gamma \Vdash[\varphi]_{\Lambda}$, then for $\Lambda^{\prime} \leq_{\star} \Lambda$, the following provides a proof of $\Gamma \Vdash[\varphi]_{\Lambda^{\prime}}$.

$$
\frac{\Gamma \Vdash[\varphi]_{\Lambda} \quad\left[\frac{}{[\varphi]_{\Lambda} \Vdash[\varphi]_{\Lambda^{\prime}}}\right.}{\Gamma \Vdash[\varphi]_{\Lambda^{\prime}}} \text { Axiom }
$$

But then, eliminating cuts implies in that case that one has a way to transform $\Pi$ into a proof of $\Gamma \Vdash[\varphi]_{\Lambda^{\prime}}$.

For this to be done, we will now introduce a operation on words which does the right manipulation on localizations.

\section{Definition 4}

Given three words $\Lambda^{\prime} \leq \Lambda \leq \Omega$, we define $\Omega / \Lambda: \Lambda^{\prime}$ as the word $\Omega_{1 \ldots l} \circ \Lambda^{\prime}$ where $l$ is the greatest integer such that $\Lambda \leq \Omega_{l+1 \ldots|\Omega|}$.

The operation works as follows : given two words $\Lambda$ and $\Omega$ such that $\Lambda \leq \Omega$ (or, stated another way, $\Lambda$ is included in $\Omega$ ), one finds the rightmost way to include $\Lambda$ in $\Omega$, "cuts" $\Omega$ at this position, and appends another word $\Lambda^{\prime}$ instead. For instance, abcdcbad/dba:a = abca, as illustrated in the following decomposition :

$$
\mathrm{abc}|\underline{\text { dcbad }} \rightsquigarrow \mathrm{abc}| \mathrm{a}
$$

Let us now express a few basic properties verified by this operation. First, given two words $\Lambda \leq \Lambda^{\prime}$, operation $\_/ \Lambda^{\prime}: \Lambda$ is $\leq$-monotonous :

$$
\Omega \leq \Omega^{\prime} \Rightarrow \Omega / \Lambda^{\prime}: \Lambda \leq \Omega^{\prime} / \Lambda^{\prime}: \Lambda
$$

If $\Lambda \leq_{\star} \Lambda^{\prime}$, then it is $\leq_{\star}$-monotonous :

$$
\Omega \leq_{\star} \Omega^{\prime} \Rightarrow \Omega / \Lambda^{\prime}: \Lambda \leq_{\star} \Omega^{\prime} / \Lambda^{\prime}: \Lambda
$$

Moreover, for $\Lambda \leq_{\star} \Lambda^{\prime}$, operation $\_/ \Lambda^{\prime}: \Lambda$ is $\leq_{\star}$-reductive :

$$
\Omega / \Lambda^{\prime}: \Lambda \leq_{\star} \Omega
$$

Finally, if $\Lambda \leq_{\star} \Lambda^{\prime}$, one can express a composition rule :

$$
\Omega \leq \Omega^{\prime} \Rightarrow \Omega^{\prime} / \Omega:\left(\Omega / \Lambda^{\prime}: \Lambda\right) \leq_{\star} \Omega^{\prime} / \Lambda^{\prime}: \Lambda
$$

As one can see, the _/_:_ operations have, considering the previous properties, some connections with the transformations functions $f_{i \mid j}$ used in the definition of representation systems, and as a consequence they are convenient tools for dealing with localizations. The next proposition shows how they can apply to proof manipulation.

\section{Proposition 4}

Given a proof $\Pi$ of a sequent $\Gamma \vdash[\varphi]_{\Lambda}$, it is possible to turn it into a proof $\Pi^{\prime}$ of $\Gamma_{/ \Lambda: \Lambda^{\prime}} \vdash[\varphi]_{\Lambda^{\prime}}$ for $\Lambda^{\prime} \leq_{\star} \Lambda$.

The notation $\Gamma_{/ \Lambda: \Lambda^{\prime}}$ corresponds to replacing each $[\psi]_{\Omega}$ in $\Gamma$ by $[\psi]_{\Omega / \Lambda: \Lambda^{\prime}}$.

Moreover, the structure of $\Pi^{\prime}$ differs only from that of $\Pi$ by the addition of some applications of the Loc-rule.

Sketch of Proof This result can be proven by induction on the size of the proof, and rely mainly on the properties of the operation _/_:_. The main rules to be examined are Loc, Cut and $\rightarrow L$.

The validity of Loc comes from the fact that for $\Lambda^{\prime} \leq_{\star} \Lambda$, then_/ $\Lambda: \Lambda^{\prime}$ is $\leq_{\star}$-monotonous.

The validit of rules Cut and $\rightarrow L$ is a consequence of the composition property : if $\Lambda^{\prime} \leq_{\star} \Lambda \leq \Omega^{\prime} \leq \Omega$, then one has $\Omega / \Omega^{\prime}:\left(\Omega^{\prime} / \Lambda: \Lambda^{\prime}\right) \leq_{\star} \Omega / \Lambda: \Lambda^{\prime}$.

\section{Corollary 4.1}

Given a proof $\Pi$ of a sequent $\Gamma \vdash[\varphi]_{\Lambda}$, it is possible to turn it into a proof $\Pi^{\prime}$ of $\Gamma \vdash[\varphi]_{\Lambda^{\prime}}$ for $\Lambda^{\prime} \leq_{\star} \Lambda$.

Moreover, the structure of $\Pi^{\prime}$ differs only from that of $\Pi$ by the addition of some applications of the Loc-rule.

Proof It is a combination of the previous proposition and the application of the Loc-rule. 


\section{Corollary 4.2}

Given a proof $\Pi$ of a sequent $\Gamma,[\varphi]_{\Lambda} \vdash[\psi]_{\Lambda}$, it is possible to turn it into a proof $\Pi^{\prime}$ of $\Gamma,[\varphi]_{\Lambda^{\prime}} \vdash[\psi]_{\Lambda^{\prime}}$ for $\Lambda^{\prime} \leq_{\star} \Lambda$.

Moreover, the structure of $\Pi^{\prime}$ differs only from that of $\Pi$ by the addition of some applications of the Loc-rule.

This corollary is the justification of our _/__: operation, since it is the central tool for "lowering" the localization of the right-side proposition of a sequent. This is necessary for achieving cut-elimination, which is our next topic.

\subsection{Cut-Elimination}

The manipulations presented in the previous section are essential to the cut-elimination, and together with the classical cut-elimination procedure $[10,22,11,9]$. The detailled specific proof for OL can be found in [3], and we just provide the theorem's statement :

\section{Theorem 5}

Given a proof $\Pi$ of a sequent $\Gamma \vdash[\varphi]_{\Lambda}$, it is possible to transform $\Pi$ into a cut-free proof $\Pi^{\prime}$ of the same sequent.

\subsection{Univ-Elimination}

Another rule which deserves close examination of the Univ-rule. The main problem with this rule is the question of meaning it can have in the case of an infinite index set $\mathcal{I}$, since this would lead to infinite proofs. Before tackling this problem, we will first show how to have a "normal" form of proofs with regards to the Univ-rule.

First, given a proof $\Pi$ of a sequent $\Gamma \Vdash[\varphi]_{\Lambda}$, it is possible to obtain a proof of $\Gamma_{\circ i} \Vdash[\varphi]_{\Lambda \circ i}$ where each localization $\Omega$ in $\Pi$ is replaced by $\Omega \circ i$. All rules except Univ is left unchanged, since it remains valid after adding $i$ on the right of the localizations. For the Univ-rule, one just has to select the $i$ th premise.

Thus, from a proof $\Pi$ of $\Gamma \Vdash[\varphi]_{\Lambda}$, one can get for each $i \in \mathcal{I}$ a proof $\Pi_{i}$ of $\Gamma_{\circ i} \Vdash[\varphi]_{\Lambda \circ i}$. It has to be noted that due to its requirements, none of those proofs contain an instance of the Univ-rule. Combining them together, one gets a proof $\Pi^{\prime}$ of $\Gamma \Vdash[\varphi]_{\Lambda}$ with the Univ-rule at the root. This manipulation can be summarized in the following proposition :

\section{Proposition 6}

Any proof $\Pi$ of a sequent $\Gamma \Vdash[\varphi]_{\Lambda}$ can be transformed so as to have a single instance of the Univ-rule, which instance is a the root.

Now, suppose that one has a proof $\Pi$ of a sequent $\Gamma_{\circ i} \Vdash$ $[\varphi]_{\Lambda \circ i}$ where $i$ appears nowhere in the sequent $\Gamma \Vdash[\varphi]_{\Lambda}$. Then, the index $i$ appears to be not relevant in $\Pi$, and one would want to simply erase it from $\Pi$. This can actually be done, and one has the following proposition :

\section{Proposition 7}

Given a proof $\Pi$ of a sequent $\Gamma_{\circ i} \Vdash[\varphi]_{\Lambda \circ i}$ such that $i$ is not present in the sequent $\Gamma \Vdash[\varphi]_{\Lambda}$, it is possible to transform $\Pi$ into a proof of $\Gamma \Vdash[\varphi]_{\Lambda}$ without changing its structure.

We have now turn to the case where the set $\mathcal{I}$ is infinite. Suppose one has a proof $\Pi$ of a sequent $\Gamma \Vdash[\varphi]_{\Lambda}$. As $\mathcal{I}$ is infinite, there exists an index $i \in \mathcal{I}$ which appears nowhere in $\Gamma \Vdash[\varphi]_{\Lambda}$. One can turn $\Pi$ into a proof $\Pi_{i}$ of $\Gamma_{\circ i} \Vdash[\varphi]_{\Lambda \circ i}$. It has to be noted that $\Pi_{i}$ does not contain the Univ-rule. Now, as $i$ appears nowhere in $\Gamma \Vdash[\varphi]_{\Lambda}$, it can be turned into a Univ-free proof of $\Gamma \Vdash[\varphi]_{\Lambda}$, using proposition 7 . This leads to the following theorem :

\section{Theorem 8}

For any sequent $\Gamma \Vdash[\varphi]_{\Lambda}$ that can be proven, there exists a proof of it with no instance of the Univ-rule.

\section{Conclusion}

In this article, we present an overview of representation systems framework and of observation logic, and present it in a multi-context approach. Then, we have introduced a sequent-calculus formulation of this logic. Due to the presence of a special modal axiom, namely T2 : $K_{i} K_{j} \varphi \rightarrow$ $K_{j} \varphi$, some addition to a "classical" formulation are needed for having the cut elimination and subformula property.

In order to solve this problem, we introduce and study the notion of localization which are labels indicating, for each proposition appearing in a proof, a "path" on the different contexts. Those labels are actually finite words defined using the indexes of contexts, and an examination of the behavior of modal operators led us to the definition of two partial orders on those words (together with an equivalence relation), and a ternary operation which permits to manipulate localizations in a convenient way for OL. With those elements, we have defined a sequent-calculus formulation of our logic, and given some basic proofs-manipulations theorems, mainly the cut- and univ- eliminations, allowing to ease and make more efficient the search of proofs.

\section{References}

[1] G. Bellin, V. de Paiva, and E. Ritter. Extended curry-howard correspondence for a basic constructive modal logic.

[2] G. Birkhoff. Lattice Theory. Colloquim Publications. American Mathematical Society, 3rd edition, 1967.

[3] O. Brunet. Étude de la connaissance dans le cadre d'observations partielles : La logique de l'observation. $\mathrm{PhD}$ thesis, Université Joseph Fourier, Grenoble (France), 2002. 
[4] O. Brunet. A modal logic for observation-based knowledge representation. In Proceedings of the IMLA'02 workshop (Intuitionnistic Modal Logic and Applications), Copenhaguen, DK, 2002.

[5] S. Buvac, V. Buvac, and I. A. Mason. Metamathematics of contexts. Fundamenta Informaticae, 23(2/3/4):263-301, 1995.

[6] P. Cousot and R. Cousot. Abstract interpretation and application to logic programs. Journal of Logic Programming, 13(2-3):103-179, 1992.

[7] M. Erné, J. Koslowski, A. Melton, and G. E. Strecker. A primer on galois connections, 1992.

[8] M. Fitting. Proof Methods for Modal and Intuitionnistic Logics, volume 169. D. Reidel Publishing, 1983.

[9] J. Gallier. Constructive logics. part i: A tutorial on proof systems and typed $\lambda$-calculi. Theoretical Computer Science, 110(2):249 - 339, 1993.

[10] G. Gentzen. Investigations into logical deduction. In M. E. Szabo, editor, The collected papers of Gerhard Gentzen, pages 68 - 128. North Holland, 1969.

[11] J.-Y. Girard. Proof Theory and Logical Complexity. Bibliopolis, 1987.

[12] F. Giunchiglia, A. Villafiorita, and T. Walsh. Theories of abstraction. AI Communications, 10(3-4):167-176, 1997.

[13] F. Giunchiglia and T. Walsh. A theory of abstraction. Artificial Intelligence, 56(2-3):323-390, October 1992.

[14] A. Heyting. Intuitionism: An Introduction. North-Holland, 1956.

[15] S. A. Kripke. A semantical analysis of modal logic I : normal modal propositional calculi. Zeitschrift für Mathematische Logik und Grundlagen der Mathematik, 9:67-96, 1963.

[16] J. McCarthy. Notes on formalizing contexts. In T. Kehler and S. Rosenschein, editors, Proceedings of the Thirteenth National Conference on Artificial Intelligence, pages 555560, Los Altos, California, 1993. Morgan Kaufmann.

[17] J. McCarthy. Approximate objetcs and approximate theories, 2000.

[18] P. P. Nayak. Representing multiple theories. In B. HayesRoth and R. Korf, editors, Proceedings of the Twelfth $\mathrm{Na}$ tional Conference on Artificial Intelligence, pages 1154 1160, Menlo Park, CA., 1994. AAAI Press.

[19] P. P. Nayak and A. Levy. A semantic theory of abstractions. In C. Mellish, editor, Proceedings of the Fourteenth International Joint Conference on Artificial Intelligence, pages 196-203. Morgan Kaufmann, 1995.

[20] O. Ore. Galois connections. Transactions of the American Mathematical Society, 55:493 - 513, 1944

[21] G. Pickert. Bemerkungen über galois-verbindungen. Archv. Math. J., 3:285-289, 1952.

[22] W. W. Tait. Normal derivability in classical logic. In J. Barwise, editor, The Syntax and Semantics of Infinitary Languages. Springer Verlag, 1968.

[23] D. van Dalen. Intuitionnistic logic. In D. Gabbay and F. Guenthner, editors, Handbook of Philosophical Logic, volume III, pages 225-340. Reidel, 1986.

$$
\begin{aligned}
& \frac{\Lambda^{\prime} \leq_{\star} \Lambda}{[\varphi]_{\Lambda} \Vdash[\varphi]_{\Lambda^{\prime}}} \text { Axiom } \quad \frac{\Lambda^{\prime} \leq \Lambda}{[\perp]_{\Lambda} \Vdash[\perp]_{\Lambda^{\prime}}} \perp \\
& \frac{\Gamma \Vdash[\psi]_{\Lambda}}{\Gamma,[\varphi]_{\Lambda} \Vdash[\psi]_{\Lambda}} \text { Weak } \frac{\Gamma,[\varphi]_{\Lambda},[\varphi]_{\Lambda} \Vdash \psi}{\Gamma,[\varphi]_{\Lambda} \Vdash \psi} \text { Contract } \\
& \frac{\Gamma \Vdash[\varphi]_{\Lambda} \quad \Delta,[\varphi]_{\Lambda^{\prime}} \Vdash \psi \quad \Lambda^{\prime} \leq_{\star} \Lambda}{\Gamma, \Delta \Vdash \psi} \mathrm{Cut} \\
& \frac{\forall i, \Gamma_{\circ i} \Vdash[\varphi]_{i}}{\Gamma \Vdash[\varphi]_{\varepsilon}} \operatorname{Univ} \quad \frac{\Gamma,[\varphi]_{\Lambda^{\prime}} \Vdash \psi \quad \Lambda^{\prime} \leq_{\star} \Lambda}{\Gamma,[\varphi]_{\Lambda} \Vdash \psi} \operatorname{Loc} \\
& \frac{\Gamma,[\varphi]_{\Lambda} \Vdash \vartheta \quad \Gamma,[\psi]_{\Lambda} \Vdash \vartheta}{\Gamma,[\varphi \vee \psi]_{\Lambda} \Vdash \vartheta} \vee L \\
& \frac{\Gamma \Vdash[\varphi]_{\Lambda}}{\Gamma \Vdash[\varphi \vee \psi]_{\Lambda}} \vee R_{1} \quad \frac{\Gamma \Vdash[\psi] \Lambda}{\Gamma \Vdash[\varphi \vee \psi]_{\Lambda}} \vee R_{2} \\
& \frac{\Gamma,[\varphi]_{\Lambda} \Vdash \vartheta}{\Gamma,[\varphi \wedge \psi]_{\Lambda} \Vdash \vartheta} \wedge L_{1} \quad \frac{\Gamma,[\psi]_{\Lambda} \Vdash \vartheta}{\Gamma,[\varphi \wedge \psi]_{\Lambda} \Vdash \vartheta} \wedge L_{2} \\
& \frac{\Gamma \Vdash[\varphi]_{\Lambda} \quad \Gamma \Vdash[\psi]_{\Lambda}}{\Gamma \Vdash[\varphi \wedge \psi]_{\Lambda}} \wedge R \\
& \frac{\Gamma \Vdash[\varphi]_{\Lambda} \quad \Gamma,[\psi]_{\Lambda} \Vdash \vartheta}{\Gamma,[\varphi \rightarrow \psi]_{\Lambda} \Vdash \vartheta} \rightarrow L \quad \frac{\Gamma,[\varphi]_{\Lambda} \Vdash[\psi]_{\Lambda}}{\Gamma \Vdash[\varphi \rightarrow \psi]_{\Lambda}} \rightarrow R \\
& \frac{\Gamma,[\varphi]_{i \circ \Lambda} \Vdash[\psi]_{\Lambda^{\prime}} \quad \Lambda^{\prime} \leq \Lambda}{\Gamma,\left[K_{i} \varphi\right]_{\Lambda} \Vdash[\psi]_{\Lambda^{\prime}}} K L \quad \frac{\Gamma \Vdash[\varphi]_{i \circ \Lambda}}{\Gamma \Vdash\left[K_{i} \varphi\right]_{\Lambda}} K R
\end{aligned}
$$

Figure 3. Sequent-Calculus Rules 